\title{
Path Integral Monte-Carlo method for relativistic quantum systems
}

\author{
Oleg Pavlovsky ${ }^{* \dagger}$ \\ Institute for Theoretical Problems of Microphysics, Moscow State University and Institute for \\ Theoretical and Experimental Physics, Moscow, Russia \\ E-mail: ovpegoa.bog.msu.ru
}

\section{Alexander Ivanov}

Department of Physics, Moscow State University, Moscow, Russia

E-mail: ivanov.asephysics.msu.ru

\section{Alexander Novoselov}

Institute for Theoretical Problems of Microphysics, Moscow State University and Institute for Theoretical and Experimental Physics, Moscow, Russia

E-mail: novoselovdgoa.bog.msu.ru

Relativistic generalization of Path Integral Monte-Carlo method has been proposed and some possible applications have been discussed.

The 32nd International Symposium on Lattice Field Theory

23-28 June, 2014

Columbia University New York, NY

\footnotetext{
* Speaker.

${ }^{\dagger}$ This work is supported in part by RFBR Grant No. 14-02-01261. Numerical calculations were performed at Supercomputing Center of the Moscow State University [9].
} 


\section{Introduction}

This work is devoted to the generalization of Path Integral Monte Carlo approach [W] in the case of the relativistic systems. There are many physical problems connected with simulations of relativistic quantum mechanical systems. Relativistic corrections play very essential role in physics of the atomic systems with heavy elements due to the strong interaction potentials. One may find the problems with simulations of relativistic quantum systems in the nuclear physics, physics of hadron structure, quark-gluon plasma and in relativistic astrophysics. Recently an another interesting application of the relativistic quantum mechanics has arisen. There are so called (Pseudo)Relativistic Condensed Matter systems and one of the must interesting example of such systems is Graphene ([[]]).

The correct formulation of the many-body relativistic quantum-mechanical problem has some well-known difficulties. The kinetic and potential part of the Hamiltonian must be invariant under Lorentz transformations. The kinetic part of the Hamiltonian can be formulated in Lorentzinvariant form relatively easy but relativistic formulation of interaction requires the quantum field theory approach in general case. In this work we have considered the problem with the instantaneous interaction in the Hamiltonian. This approximation works very well in Relativistic Quantum Chemistry and in Physics of the (Pseudo)Relativistic Condensed Matter systems like Graphene.

In our work we have discussed the basic concepts of the relativistic generalization of the Path Integral approach. The main observation is that in relativistic case the statistical weight of the "Path" is not an exponent of some classical action as it is in the case of the non-relativistic systems due to the well-known Feynman-Kac formula. In spite of this fact, the relativistic statistical weight of the "Path" is well defined positive function and can be easily used in numerical calculations. In this work we test our approach on the simple academic problem - Relativistic Oscillator problem. This system gives us good opportunity for testing of the Relativistic Path Integral Monte-Carlo approach because one can study this system by using of standard Schrodinger equation approach in the momentum space. We will compare the results of these two approaches.

\section{Non-relativistic and ultra-relativistic limits for relativistic quantum particles}

Let us consider the quantum system with following Hamiltonian

$$
H=T(p)+V(q)=\sqrt{p^{2}+m^{2}}+V(q),
$$

where $q$ and $p$ is the coordinate and the momentum of the particle. $T(p)$ is the kinetic energy with rest mass and $V(q)$ is the potential energy.

In non-relativistic limit $m^{2} \gg p^{2}$ one gets

$$
T(p)=\sqrt{p^{2}+m^{2}}=m\left(1+\frac{p^{2}}{2 m^{2}}+O\left(\left(\frac{p}{m}\right)^{4}\right)\right) \approx m+\frac{p^{2}}{2 m},
$$

Typical values of momentum $p$ are dependent on the potential $V(q)$. If the interaction is strong, the values of momentum $p$ can be very large. In this case we are not able to use the non-relativistic Hamiltonian and relativistic corrections are very essential. 
As an example, in our work we have considered the Harmonic oscillator potential

$$
V(q)=\frac{1}{2} m \omega^{2} q^{2} .
$$

In non-relativistic case the virial theorem gives us the following relation between kinetic and potential energy

$$
\left\langle\frac{p^{2}}{2 m}\right\rangle=\left\langle\frac{1}{2} m \omega^{2} q^{2}\right\rangle \text { or }\langle T(p)-m\rangle=\langle V(q)\rangle
$$

Using this expression we can formulate non-relativistic condition for this system in terms of $m$ and $\omega$ :

$$
\left\langle p^{2}\right\rangle \sim m \omega \Rightarrow m \gg \omega .
$$

It means that in non-relativistic case we consider heavy particles and soft potentials.

Similarly let us consider ultra-relativistic limit $\left(\left\langle p^{2}\right\rangle \gg m^{2}\right)$. The kinetic energy in this case is follows:

$$
T(p)=\sqrt{p^{2}+m^{2}}=|p|\left(1+\frac{m^{2}}{2 p^{2}}+O\left(\left(\frac{m}{p}\right)^{4}\right)\right) \approx|p| .
$$

Let us consider the Hamiltonian in zero order approximation:

$$
H=|p|+\frac{1}{2} m \omega^{2} q^{2}
$$

In this case one can solve Schrodinger equation for this Hamiltonian in momentum representation and find energy of ground state and corresponding wave function. The virial theorem for this Hamiltonian gives us the relation between kinetic and potential energy

$$
\langle T(p)\rangle=2\langle V(q)\rangle=\frac{2 \lambda_{0}}{3}\left(m \omega^{2}\right)^{1 / 3},
$$

where $\lambda_{0}=0.808617 \ldots$. One obtains that $\langle|p|\rangle \sim\left(m \omega^{2}\right)^{1 / 3}$ and we have opposite expression for mass and frequency for ultra-relativistic case:

$$
\omega \gg m \text {. }
$$

So, ultra-relativistic limit in this problem is reached in the case of small mass and strong potentials.

\section{Path integral approach for the relativistic quantum particles}

Relativistic generalization of the Path Integral approach for quantum mechanical systems has a long history [ [B]], [四]. Today this approach becoming more and more popular and find its application in high-energy physics [5], [6].

Now let us consider relativistic generalization of the Path Integral approach. Matrix element of density matrix in the position representation is

$$
\rho\left(q, q^{\prime} ; \beta\right)=\left\langle q\left|e^{-\beta H}\right| q^{\prime}\right\rangle=\int \ldots \int d q_{1} d q_{2} \ldots d q_{N-1} \rho\left(q, q_{1} ; \tau\right) \rho\left(q_{1}, q_{2} ; \tau\right) \ldots \rho\left(q_{N-1}, q^{\prime} ; \tau\right) .
$$


Operator of kinetic energy is diagonal in the momentum representation and operator of potential energy is diagonal in the position representation. We can separate kinetic and potential energy if $\tau$ is small:

$$
\rho\left(q_{0}, q_{2} ; \tau\right) \approx \int d q_{1}\left\langle q_{0}\left|e^{-\tau T}\right| q_{1}\right\rangle\left\langle q_{1}\left|e^{-\tau V}\right| q_{2}\right\rangle .
$$

Potential energy is diagonal in this representation and for kinetic energy part one obtains

$$
\left\langle q_{0}\left|e^{-\tau T}\right| q_{1}\right\rangle=\int d p d p^{\prime} \delta\left(p-p^{\prime}\right)\left\langle q_{0} \mid p\right\rangle\left\langle p^{\prime} \mid q_{1}\right\rangle e^{-T(p) \tau}=\int \frac{d p}{2 \pi} e^{-T(p) \tau-i p\left(q_{0}-q_{1}\right)} .
$$

So to build the Path Integral we must take this integral over momentum. One can calculate integral over momentum with $T(p)=\sqrt{p^{2}+m^{2}}$

$$
\left\langle q_{0}\left|e^{-\tau T}\right| q_{1}\right\rangle=\frac{m \tau}{\pi \sqrt{\tau^{2}+\left(q_{1}-q_{0}\right)^{2}}} K_{1}\left(m \sqrt{\tau^{2}+\left(q_{1}-q_{0}\right)^{2}}\right),
$$

where $K_{1}(*)$ is modified Bessel function of first order. The general expression for matrix element is the following

$$
\rho\left(q^{\prime \prime}, q^{\prime} ; \tau\right)=\left\langle q^{\prime \prime}\left|e^{-\tau(T(p)+V(q))}\right| q^{\prime}\right\rangle=\frac{m}{\pi \sqrt{1+\left(\frac{q^{\prime \prime}-q^{\prime}}{\tau}\right)^{2}}} K_{1}\left[m \tau \sqrt{1+\left(\frac{q^{\prime \prime}-q^{\prime}}{\tau}\right)^{2}}\right] e^{-\tau V\left(q^{\prime}\right)} .
$$

One can find the multi-dimensional generalization of the expression (2.])

$$
\rho\left(q^{\prime \prime}, q^{\prime} ; \tau\right)=\left(\frac{m \tau}{\pi \sqrt{\tau^{2}+\left(\mathbf{q}^{\prime \prime}-\mathbf{q}^{\prime}\right)^{2}}}\right)^{(d+1) / 2} \frac{K_{(d+1) / 2}\left(m \sqrt{\tau^{2}+\left(\mathbf{q}^{\prime \prime}-\mathbf{q}^{\prime}\right)^{2}}\right)}{(2 \tau)^{(d-1) / 2}} \exp \left(-\tau V\left(q^{\prime}\right)\right)
$$

where $d$ is the space dimensions.

Unfortunately in this relativistic case we have no interpretation of expressions (ㅁ] and ([2]) in terms of some classical action as we have in non-relativistic case in Feynman-Kac formula. Nevertheless, this statistical weighs of the "Path" are the positively definite values. It means that we can use these expressions for the construction of the Path Integral Monte-Carlo Algorithms.

\section{Path Integral Monte-Carlo Metropolis Algorithm}

For Path Integral Monte-Carlo Metropolis algorithm we should know part of the density matrix which corresponds to fixed point $q_{i}$. Discussion about this method and all proofs one can find in [U]. Using (R. DI) we can write

$$
\pi\left(q_{i}\right)=\frac{m^{2} K_{1}\left[m \tau \sqrt{1+\left(\frac{q_{i}-q_{i-1}}{\tau}\right)^{2}}\right] K_{1}\left[m \tau \sqrt{1+\left(\frac{q_{i+1}-q_{i}}{\tau}\right)^{2}}\right]}{\pi^{2} \sqrt{1+\left(\frac{q_{i}-q_{i-1}}{\tau}\right)^{2}} \sqrt{1+\left(\frac{q_{i+1}-q_{i}}{\tau}\right)^{2}}} e^{-\tau V\left(q_{i}\right)} .
$$

So to calculate Path Integral we construct Markov chain which has equilibrium state for fixed $q_{i}$ proportional to $\pi\left(q_{i}\right)$. In Metropolis algorithm we can split transition probability

$$
P\left(q_{i} \rightarrow q_{i}^{\prime}\right)=T\left(q_{i} \rightarrow q_{i}^{\prime}\right) A\left(q_{i} \rightarrow q_{i}^{\prime}\right)
$$




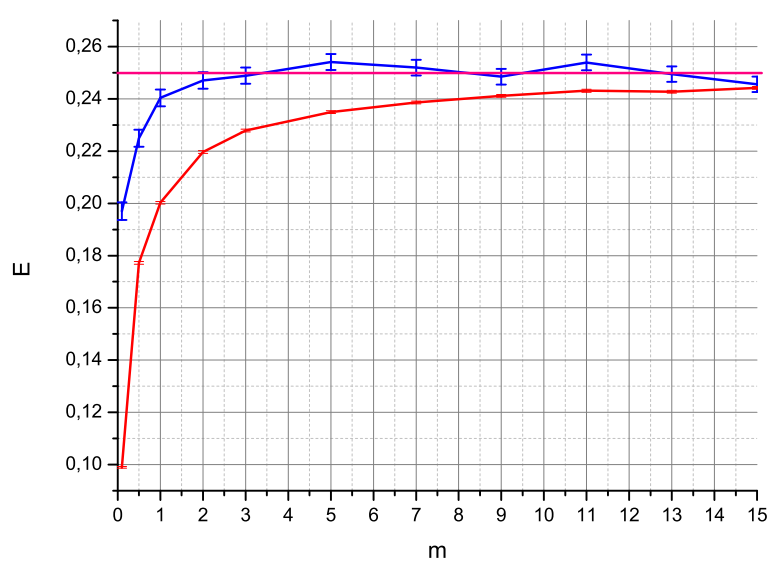

Figure 1: PIMC simulations for kinetic (blue line) and potential (red line) energy of the Relativistic Oscillator ground state. $\omega=1$.

where $T\left(q_{i} \rightarrow q_{i}^{\prime}\right)$ is sampling distribution and $A\left(q_{i} \rightarrow q_{i}^{\prime}\right)$ is acceptance probability

$$
A\left(q_{i} \rightarrow q_{i}^{\prime}\right)=\min \left[1, \frac{T\left(q_{i}^{\prime} \rightarrow q_{i}\right) \pi\left(q_{i}^{\prime}\right)}{T\left(q_{i} \rightarrow q_{i}^{\prime}\right) \pi\left(q_{i}\right)}\right]
$$

If we know density matrix in the position representation, we can calculate expressions for average of any observables. The expression for kinetic energy in the relativistic case is the following (see [8] ):

$$
\langle T(p)\rangle=\left\langle\frac{m \tau}{\sqrt{\tau^{2}+(\Delta q)^{2}}} \frac{K_{0}\left(m \sqrt{\tau^{2}+(\Delta q)^{2}}\right)}{K_{1}\left(m \sqrt{\tau^{2}+(\Delta q)^{2}}\right)}+\frac{\tau^{2}-(\Delta q)^{2}}{\tau\left(\tau^{2}+(\Delta q)^{2}\right)}\right\rangle .
$$

And total energy can be calculated as follows:

$$
\langle E(p, q)\rangle=\langle T(p)+V(q)\rangle .
$$

\section{One dimensional test: Relativistic Oscillator}

For the testing of the Relativistic PIMC approach let us consider the simple academic problem - Relativistic Oscillator in one space dimension. This problem can be studied by using of the standard Schrodinger equation approach in the momentum space and we will compare the results of this two approaches.

On Fig. 1 one can see the results of the PIMC simulations for kinetic and potential energy of the Relativistic Oscillator ground state. For these simulations we have used 1500 statistically independent configurations. In this calculation we have fixed the value of $\omega=1$ and have changed the value of mass $m$. In this case the ultra-relativistic limit is reached at $m \ll 1$ while non-relativistic limit is reached at $m \gg 1$ correspondingly.

The virial theorem predicts the following expressions for kinetic and potential energy

$$
\langle T-m\rangle_{\text {non-rel }}=\langle V\rangle_{\text {non-rel }}=0.25
$$




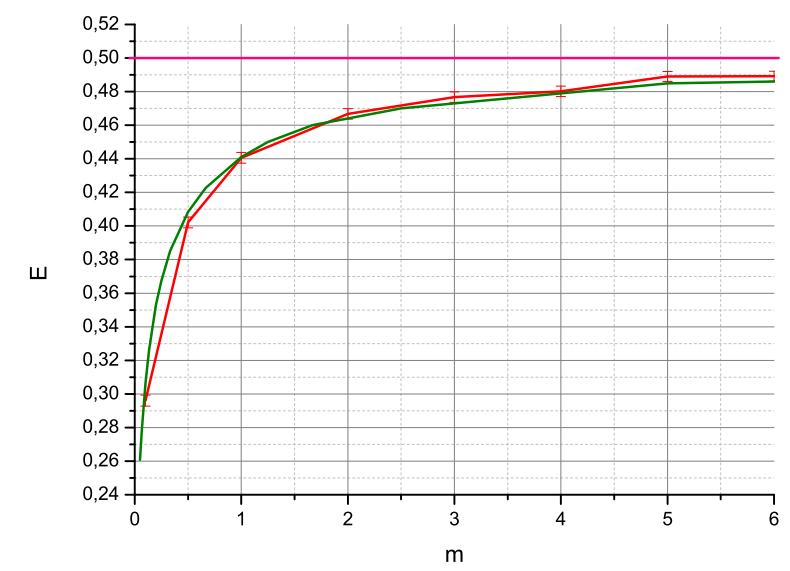

Figure 2: PIMC simulations for total energy of the Relativistic Oscillator ground state. $\omega=1$. The green line is the results of Schrodinger equation approach.

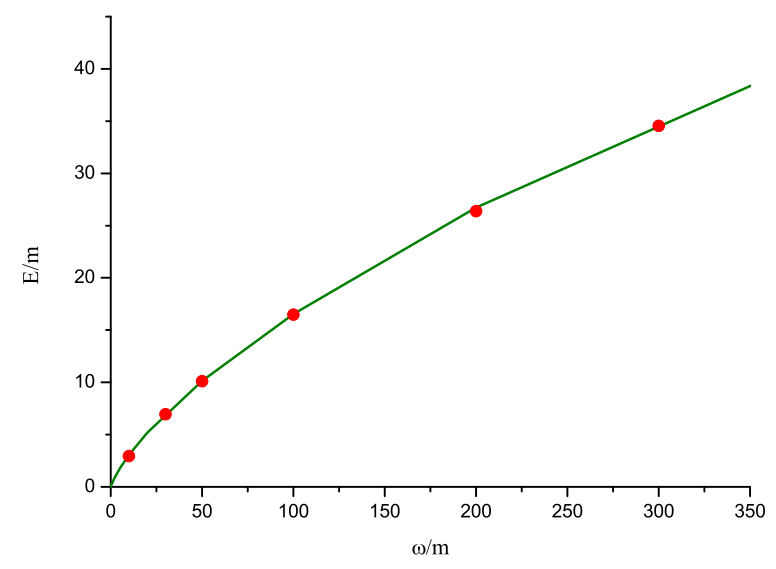

Figure 3: Total energy of the Relativistic Oscillator ground state for different $\omega$ and $m$. The green line is the result of Schrodinger equation approach. Red points are the results of PIMC approach.

at the non-relativistic limit and

$$
\langle T\rangle_{\text {ultra-rel }}=\langle V\rangle_{\text {ultra-rel }} / 2
$$

at the ultra-relativistic limit. Our PIMC results are in very good agreement with both these expressions.

On Fig. 2 one can find the results of PIMC simulations of the total energy of the ground state at $\omega=1$. In non-relativistic limit the energy of the ground state equals to $\omega / 2=0.5$. The green line is the result of Schrodinger equation approach. One can see that PIMC results are in the good agreement the results of the Schrodinger equation approach.

On Fig. 3 one can see the test of the PIMC method for strong potentials. In this calculation 
one can fix the value of the mass (for example, $m=1$ ) and change the value of $\omega$. The green line is again the results of Schrodinger equation approach and red points are the PIMC results. In this analyzing we have found out that PIMC method can be applied for very strong potentials.

\section{Discussion of the results and conclusion}

The main goal of our work is to construct relativistic generalization of the PIMC method. This method we plan to use for investigation of the properties of the relativistic quantum systems with instantaneous interactions between the particles. Last fact gives us possibility to avoid the problem with correctness of the many-body quantum-mechanical interaction. For example, this approach could be used for studies the thermodynamical and transport properties of graphene which are very essential for future technological application. Of course, there are many interesting applications one can find in Relativistic Quantum Chemistry too.

For testing of our approach, we study simple one-dimensional system with quadratic external potential - relativistic oscillator. This system gives us the good possibility for testing our approach because just relativistic oscillator can be studied by using Schrodinger equation in momentum space. The comparison the results of these two approaches have shown that our relativistic generalization of PIMC method can be used for investigation of quantum systems which contain the relativistic particles.

\section{References}

[1] D. M. Ceperley Path integrals in the theory of condensed helium, Rev.Mod.Phys. 67 (1995) 279-355

[2] K. S. Novoselov, A. K. Geim, S. V. Morozov, D. Jiang, Y. Zhang, S. V. Dubonos, I. V. Grigorieva, and A. A. Firsov, Electric Field Effect in Atomically Thin Carbon Films, Science 306 (2004) 666.

[3] P. P. Fiziev, Relativistic Hameltonians wit square-roofs in the Path Integral formaism, Theor. Math. Phys. 62 (1985) 186.

[4] I. H. Redmount and W.-M. Suen, Path integration in relativistic quantum mechanics, Int. J. Mod. Phys. A 08 (1993) 1629.

[5] V.S. Filinov, Yu.B. Ivanov, V.E. Fortov, M. Bonitz, and P.R. Levashov, Color path-integral Monte-Carlo simulations of quark-gluon plasma: Thermodynamic and transport properties, Phys.Rev. C 87 (2013) 035207.

[6] V.S. Filinov, M. Bonitz, Y.B. Ivanov, M. Ilgenfritz, and V.E. Fortov, Thermodynamics of the quark-gluon plasma at finite chemical potential: color path integral Monte Carlo results, arXiv: 1408.5714

[7] M. Creutz and B.A. Freedman, A statistical approach to quantum mechanics, Ann. Phys. 132 (1981) 427.

[8] H. J. Rothe Lattice Gauge Theories: An Introduction (3rd Edition) (World Scientific Lecture Notes in Physics). World Scientific Publishing Co. Pte. Ltd. 2005

[9] Vl.V. Voevodin, S.A. Zhumatiy, S.I. Sobolev, A.S. Antonov, P.A. Bryzgalov, D.A. Nikitenko, K.S. Stefanov, Vad.V. Voevodin Practice of "Lomonosov" Supercomputer, Open Systems J. - Moscow: Open Systems Publ., 2012, no.7. 